\title{
Impact of Sarcopenia on Chemotherapy-Triggered Exacerbation of Interstitial Lung Disease in Patients With Non-Small Cell Lung Cancer
}

Ryota Kikuchi ( $\square$ reo1129@tokyo-med.ac.jp )

Tokyo Medical University Hospital

Hiroyuki Takoi

Tokyo Medical University Hospital

Mayuko Ishiwari

Tokyo Medical University Hospital

Kazutoshi Toriyama

Tokyo Medical University Hospital

Yuta Kono

Tokyo Medical University Hospital

\section{Yuki Togashi}

Tokyo Medical University Hospital

Shinji Abe

Tokyo Medical University Hospital

\section{Research Article}

Keywords: non-small cell lung cancer, interstitial lung disease, psoas muscle index, sarcopenia, exacerbation, chemotherapy, prognosis

Posted Date: July 1st, 2021

DOI: https://doi.org/10.21203/rs.3.rs-658884/v1

License: (c) (1) This work is licensed under a Creative Commons Attribution 4.0 International License.

Read Full License

Version of Record: A version of this preprint was published at Thoracic Cancer on December 28th, 2021. See the published version at https://doi.org/10.1111/1759-7714.14294. 


\section{Abstract}

Background: While recent evidence has suggested that sarcopenia could predict chemotoxicity, its association with chemotherapy-triggered interstitial lung disease (ILD) exacerbations has yet to be investigated. Thus, the present study sought to determine whether sarcopenia could predict ILD exacerbations and overall survival (OS) in patients with ILD-complicated non-small cell lung cancer (NSCLC).

Methods: From January 2010 to July 2020, 74 patients with ILD-complicated NSCLC who received chemotherapy were retrospectively investigated. After categorizing patients according to the presence or absence of sarcopenia based on the psoas muscle index, ILD exacerbation rates and OS were evaluated.

Results: Among the included patients, 39 were included in the sarcopenia group. Moreover, 17 (22.9\%) patients developed ILD exacerbations, with the sarcopenia and no-sarcopenia groups having an exacerbation rate of $33.3 \%$ and $11.4 \%$, respectively $(p=0.025)$. Multivariate analysis identified sarcopenia as an independent predictor of ILD exacerbations $(p=0.039)$. Furthermore, patients with sarcopenia demonstrated a significantly shorter median OS compared to those without the same (9.2 vs. 13.3 months; $p=0.029$ ).

Conclusions: Sarcopenia predicted chemotherapy-triggered ILD exacerbation and OS in patients with ILDcomplicated NSCLC, suggesting its utility in determining treatment approaches.

Classifications: Pneumonology

\section{Introduction}

Follow-up studies have found that $10-20 \%$ of patients with interstitial lung disease (ILD) had lung cancer ${ }^{1,2}$. In particular, studies limited to idiopathic pulmonary fibrosis (IPF), which accounts for more than $50 \%$ of idiopathic ILD, have shown that $12.7-31.3 \%$ of patients with IPF presented with lung cancer $^{1-4}$. Although several recent single-group trials have investigated patients with lung cancer who have pre-existing ILD 5,6 , such patients have largely been excluded from most clinical trials involving chemotherapy development given the risk for treatment associated exacerbation of ILD. Therefore, insufficient evidence has been available for the treatment and prognosis of such patients.

Studies have shown that lung cancer with ILD has a poor prognosis because of the risk for chemotherapy-triggered exacerbation of ILD $2,7,8$. ILD exacerbation rate at the time of chemotherapy is $27-34.9 \%^{9-11}$, with exacerbation-related mortality rate ranges from $17.6 \%$ to as high as $64.2 \% 2,12,13$. Therefore, predicting ILD exacerbation may be beneficial in improving the prognosis of lung cancer with ILD. Although studies have already identified usual interstitial pneumonia patterns in high-resolution computed tomography (HRCT), low vital capacity, Gender-Age-Physiology score, and Glasgow prognostic score (GPS) as predictors of exacerbation $9,10,12,14,15$, clinically effective factors that can reliably predict exacerbations have yet to be elucidated. 
Sarcopenia is a syndrome characterized by progressive and systemic loss of skeletal muscle mass and strength ${ }^{16}$. Incidentally, recent reports have identified low skeletal muscle mass measured via computed tomography (CT) as a poor prognosis factor for lung cancer ${ }^{17-20}$ and ILD ${ }^{21-25}$. Additionally, evidence has shown that sarcopenia was associated with chemotherapeutic dose-limiting toxicity and severe toxicity events in patients with various cancers ${ }^{20,26,27}$. Cortellini et al. reported that among patients with lung cancer, those who had sarcopenia exhibited significantly higher hematological toxicity rates compared to those who did not. However, no study has yet investigated the relationship between chemotherapyinduced lung damage or ILD exacerbation and skeletal muscle mass or sarcopenia.

Although the etiology of sarcopenia remains unclear, chronic inflammation and malnutrition due to regulation of myokine production have been considered causative factors ${ }^{16}$. Our group had previously reported that GPS, which is calculated using serum C-reactive protein and albumin, can predict the prognosis and exacerbation of ILD in patients with ILD-complicated lung cancer ${ }^{14,15}$, suggesting the potential involvement of inflammation and nutrition in the pathophysiology of this disease. Therefore, the present study sought to determine whether the psoas major muscle area at the third lumbar vertebral level on CT, which has been used as an index that reflects the entire skeletal muscle mass ${ }^{28}$, could potentially be a biomarker for predicting exacerbation of ILD and overall survival (OS) in patients with ILD-complicated lung cancer.

\section{Results}

\subsection{Patient characteristics}

Baseline characteristics of the study subjects are provided in Table 1. Among the included patients, 17 $(23 \%)$ and 57 (77\%) were female and male, respectively, with a median age of 71 (range 66-75). Over 90.5\% of the patients were diagnosed with an Eastern Cooperative Oncology Group (ECOG) performance status (PS) of 0 or 1 . Median body mass index (BMI) was $21.6 \mathrm{~kg} / \mathrm{m}^{2}$ (range $19.0-23.3 \mathrm{~kg} / \mathrm{m}^{2}$ ) and 23.8 $\mathrm{kg} / \mathrm{m}^{2}$ (range $21.5-25.5 \mathrm{~kg} / \mathrm{m}^{2}$ ) for female and male patients, respectively. Moreover, 41 patients (55.4\%) were histologically diagnosed with adenocarcinoma, whereas 58 patients $(78.4 \%)$ were diagnosed with stage IV or recurrence. The median serum lactate dehydrogenase and Krebs von den Lungen 6 levels were $221.5 \mathrm{U} / \mathrm{L}$ and $681.5 \mathrm{U} / \mathrm{mL}$, respectively. The median predicted forced vital capacity (FVC) and \% predicted diffuse capacity of the lung for carbon monoxide were $103.0 \%$ and $67.9 \%$, respectively. The median psoas muscle index (PMI) was $4.48 \mathrm{~cm}^{2} / \mathrm{m}^{2}$ (range $3.70-5.43 \mathrm{~cm}^{2} / \mathrm{m}^{2}$ ) and $6.23 \mathrm{~cm}^{2} / \mathrm{m}^{2}$ (range $5.33-7.14 \mathrm{~cm}^{2} / \mathrm{m}^{2}$ ) for female and male patients, respectively. 
Table 1

Patient characteristics

\section{Number (\%) or Median (Range)}

Total patients

Age (years)

$\operatorname{Sex}(\%)$

Female

Male

Performance status (\%)

0,1

2-4

BMI $\left(\mathrm{kg} / \mathrm{m}^{2}\right)$

Female

Male

Histology (\%)

Adenocarcinoma

Non - adenocarcinoma

Clinical stage (\%)

III

IV or recurrence

LD (U/L)

$\mathrm{KL}-6(\mathrm{U} / \mathrm{mL})$

$\%$ predicted FVC (\%)

$\%$ predicted DLCO (\%)

$\mathrm{PMI}\left(\mathrm{cm}^{2} / \mathrm{m}^{2}\right)$

Female

Male

$\mathrm{BMI}$, body mass index; LD, lactate dehydrogenase; KL-6, Krebs von den Lungen 6; FVC, forced vital capacity; DLCO, diffuse capacity of the lung for carbon monoxide; PMI, psoas muscle index.

$74(100 \%)$

$71(66-75)$

$17(23.0 \%)$

$57(77.0 \%)$

$67(90.5 \%)$

$7(9.5 \%)$

$23.1(20.9-25.0)$

$21.6(19.0-23.3)(n=17)$

$23.8(21.5-25.5)(n=57)$

$41(55.4 \%)$

$33(44.6 \%)$

$16(21.6 \%)$

$58(78.4 \%)$

$221.5(198-269.2)$

681.5 (472.5-1029.5)

103.0 (84.8-118.0)

$67.9(53.3-88.2)(n=28)$

$5.91(5.02-6.65)$

$4.48(3.70-5.43)(\mathrm{n}=17)$

$6.23(5.33-7.14)(n=57)$ 


\subsection{Correlation between sarcopenia and clinicopathological parameters}

Based on the PMI cutoffs for Asian adults, 39 (52.7\%) patients were diagnosed with sarcopenia. Table 2 summarizes the baseline clinicopathological characteristics of the patients according to sarcopenia status. Male patients were more likely to have sarcopenia compared to female patients. No significant differences were noted between both groups except for sex $(p=0.028)$, BMI of males $(p=0.044)$, and PMI $(p<0.001$ and $p<0.001$ in females and males, respectively) 
Table 2

Clinical characteristics stratified according to sarcopenia status

Number (\%) or Median (Range)

p-value

\begin{tabular}{llll} 
& Nonsarcopenia $(\mathrm{n}=35)$ & Sarcopenia $(\mathrm{n}=39)$ & \\
Age (years) & $69(64-74)$ & $72(69-76)$ & 0.054 \\
Sex $(\%)$ & & & 0.028 \\
Female & $12(34.3 \%)$ & $5(12.8 \%)$ & \\
Male & $23(65.7 \%)$ & $34(87.2 \%)$ & 0.583 \\
Performance status $(\%)$ & & & \\
0,1 & $31(88.6 \%)$ & $36(92.3 \%)$ & 0.258 \\
$2-4$ & $4(11.4 \%)$ & $3(7.7 \%)$ & 0.799 \\
BMl $\left(\mathrm{kg} / \mathrm{m}^{2}\right)$ & $23.5(20.2-25.7)$ & $23.1(21.0-24.2)$ & \\
Female & $20.9(19.1-23.6)$ & $21.6(17.7-23.3)$ & 0.044 \\
& $(\mathrm{n}=12)$ & $(\mathrm{n}=5)$ & \\
\hline Male & $24.4(22.9-26.5)$ & $23.1(21.0-24.7)$ & $(\mathrm{n}=34)$ \\
& $(\mathrm{n}=23)$ & &
\end{tabular}

Histology (\%)

Adenocarcinoma

$23(65.7 \%)$

$18(46.2 \%)$

0.091

Non - adenocarcinoma

$12(34.3 \%)$

$21(53.8 \%)$

\section{Clinical stage (\%)}

III

IV or recurrence

LD (U/L)

$\mathrm{KL}-6(\mathrm{U} / \mathrm{mL})$

\% predicted FVC (\%)

$\%$ predicted DLCO (\%)
7 (20.0\%)

$28(80.0 \%)$

223 (202-267)

628 (460-1549)

101.4 (86.5-114.8)

$63.7(42.3-84.9)$

$(\mathrm{n}=12)$

$6.71(5.59-7.38)$
$9(23.1 \%)$

0.748

30 (76.9\%)

220 (197-270)

0.641

684 (476-963)

0.603

$103.0(82.8-118.6)$

0.984

70.4 (62.6-96.7)

0.260

$(\mathrm{n}=16)$

$5.35(4.62-6.08)$ $<0.001$

PMI $\left(\mathrm{cm}^{2} / \mathrm{m}^{2}\right)$

BMI, body mass index; LD, lactate dehydrogenase; KL-6, Krebs von den Lungen 6; FVC, forced vital capacity; DLCO, diffuse capacity of the lung for carbon monoxide; PMI, psoas muscle index. 


\begin{tabular}{|llll|}
\hline \multicolumn{3}{|c|}{ Number (\%) or Median (Range) } & p-value \\
\hline Female & $4.96(4.24-5.70)$ & $3.44(2.18-3.70)$ & $<0.001$ \\
& $(\mathrm{n}=12)$ & $(\mathrm{n}=5)$ & $<0.001$ \\
\hline Male & $7.29(6.71-7.60)$ & $5.41(5.09-6.11)$ & \\
& $(\mathrm{n}=23)$ & $(\mathrm{n}=34)$ & \\
\hline $\begin{array}{l}\text { BMI, body mass index; LD, lactate dehydrogenase; KL-6, Krebs von den Lungen 6; FVC, forced vital } \\
\text { capacity; DLCO, diffuse capacity of the lung for carbon monoxide; PMI, psoas muscle index. }\end{array}$ \\
\hline
\end{tabular}

\subsection{Exacerbation of chemotherapy-triggered ILD}

Among the 74 patients, 17 (22.9\%) were diagnosed with chemotherapy-triggered exacerbation ILD. Thereafter, risk factors for ILD exacerbation were evaluated (Table 3). Univariate logistic regression analysis showed that the incidence ILD exacerbation was significantly associated with \% predicted FVC [odds ratio (OR), 0.96; $95 \%$ confidence interval $(\mathrm{Cl}), 0.94-0.99 ; p=0.035$ ] and sarcopenia (OR, 3.87; $95 \%$ $\mathrm{Cl}, 1.12-13.33 ; p=0.032)$. Multivariate analysis identified age $(\mathrm{OR}, 0.89 ; 95 \% \mathrm{Cl}, 0.80-0.98, p=0.026), \%$ predicted FVC (OR, 0.96; 95\% Cl, 0.93-0.99; $p=0.026)$, and sarcopenia (OR, 6.39; 95\% Cl, 1.09-37.26; $p=$ 0.039) as significant independent predictive factors for ILD exacerbation after adjusting for age, sex, BMI, $\%$ predicted FVC, and sarcopenia. 
Table 3

Factors associated with chemotherapy-triggered exacerbation of ILD following univariate and multivariate analyses.

\begin{tabular}{|c|c|c|c|c|c|c|}
\hline \multirow[t]{2}{*}{ Factors } & \multicolumn{3}{|c|}{ Univariate analysis } & \multicolumn{3}{|c|}{ Multivariate analysis } \\
\hline & OR & $95 \% \mathrm{Cl}$ & $\begin{array}{l}p- \\
\text { value }\end{array}$ & OR & $95 \% \mathrm{Cl}$ & $p$-value \\
\hline Age, per year increment & 0.93 & $\begin{array}{l}0.86- \\
1.01\end{array}$ & 0.105 & 0.89 & $\begin{array}{l}0.80- \\
0.98\end{array}$ & 0.026 \\
\hline Sex (female vs. male) & 0.64 & $\begin{array}{l}0.18- \\
2.17\end{array}$ & 0.474 & 0.71 & $\begin{array}{l}0.11- \\
4.31\end{array}$ & 0.712 \\
\hline $\begin{array}{l}\text { Performance status } \\
(0,1 \text { vs. } 2-4)\end{array}$ & 1.38 & $\begin{array}{l}0.24- \\
7.88\end{array}$ & 0.712 & & & \\
\hline BMI, per $\mathrm{kg} / \mathrm{m}^{2}$ increment & 0.92 & $\begin{array}{l}0.77- \\
1.11\end{array}$ & 0.417 & 0.98 & $\begin{array}{l}0.79- \\
1.22\end{array}$ & 0.921 \\
\hline $\begin{array}{l}\text { Histology (adenocarcinoma vs. } \\
\text { nonadenocarcinoma) }\end{array}$ & 0.60 & $\begin{array}{l}0.19- \\
1.86\end{array}$ & 0.382 & & & \\
\hline Clinical stage (III vs. IV or Recurrence) & 1.37 & $\begin{array}{l}0.34- \\
5.54\end{array}$ & 0.651 & & & \\
\hline LD, per U/L increment & 1.00 & $\begin{array}{l}0.99- \\
1.01\end{array}$ & 0.072 & & & \\
\hline $\mathrm{KL}-6$, per $\mathrm{U} / \mathrm{mL}$ increment & 1.00 & $\begin{array}{l}1.00- \\
1.00\end{array}$ & 0.353 & & & \\
\hline$\%$ predicted FVC, per \% increment & 0.96 & $\begin{array}{l}0.94- \\
0.99\end{array}$ & 0.035 & 0.96 & $\begin{array}{l}0.93- \\
0.99\end{array}$ & 0.026 \\
\hline $\begin{array}{l}\% \text { predicted DLCO, per } \% \text { increment }(n= \\
28)\end{array}$ & 0.73 & $\begin{array}{l}0.95- \\
1.03\end{array}$ & 0.993 & & & \\
\hline Sarcopenia (no vs. yes) & 3.87 & $\begin{array}{l}1.12- \\
13.33\end{array}$ & 0.032 & 6.39 & $\begin{array}{l}1.09- \\
37.26\end{array}$ & 0.039 \\
\hline
\end{tabular}

\subsection{Prognosis}

Kaplan-Meier analysis was conducted to examine the impact of sarcopenia on survival. Figure 1 shows that those who had sarcopenia demonstrated a significantly worse OS compared to those who did not (9.2 vs. 13.3 months; $p=0.029$ ). The chemotherapy regimens are detailed in Supplementary Table S1. The combination of carboplatin and paclitaxel with/without bevacizumab was the most frequently used first-line chemotherapy. After comparing chemotherapy regimens between patients who did and did not have sarcopenia, no significant differences were found (Supplemental Table S2). 


\section{Discussion}

To the best of our knowledge, this is the first study to evaluate and explore the impact of sarcopenia in patients with NSCLC and pre-existing ILD by quantitatively measuring PMI. The present study showed a correlation between sarcopenia and the incidence of ILD exacerbation and prognosis, with sarcopenia having been identified as an independent predictor of chemotherapy-triggered exacerbation of ILD after adjusting for established prognostic factors of NSCLC and ILD. Furthermore, our findings showed that patients with sarcopenia exhibited a significantly lower median OS compared to those without it. The aforementioned results suggest the importance of monitoring PMI in clinical settings. Furthermore, the management of sarcopenia may prevent exacerbations of ILD and improve prognosis, highlighting a novel therapeutic target among such patients.

Although several studies have investigated the relationship between ILD prognosis and skeletal muscle mass $^{21-24}$, only one has thus far examined the relationship between ILD exacerbations and skeletal muscle mass. Awano et al. reported that the cross-sectional area of the erector spinae muscles measured at the 12th thoracic vertebral body level predicted the prognosis of patients with IPF but did not contribute to IPF exacerbation ${ }^{23}$, which diffed from the results presented herein. This could have been attributed to our use of the psoas major muscle instead of the erector spinae muscle. Although all previous studies on ILD have used the erector spinae muscles, lung cancer studies often use the psoas major muscle at the third lumbar spine level ${ }^{17-20,29}$ given that the muscle group found at the cranial level $5 \mathrm{~cm}$ from the fourth-fifth lumbar vertebra has been considered the most accurate estimate of whole-body muscle mass $^{30}$. The PMl used in the present study, which can be easily calculated from the psoas major muscle cross-sectional area and height, has been suggested to have a strong correlation with the skeletal muscle index ${ }^{28}$. Moreover, criteria for low skeletal muscle mass among Asians $\left(6.36\right.$ and $3.92 \mathrm{~cm}^{2} / \mathrm{m}^{2}$ for men and women, respectively) have been widely used in lung cancer research as indicators of sarcopenia ${ }^{17,18,29}$. The present study, which has been the first to utilize the PMI for ILD, found that sarcopenia can predict chemotherapy-triggered ILD exacerbation. As such, we believe that sarcopenia evaluation based on the psoas major muscle may become the standard in the future studies investigating ILD.

ILD exacerbations observed herein had been limited to antineoplastic causes, which could also be another point of difference from a previous report ${ }^{23}$. Although various chemotherapy regimens had been included, patients with ILD who received the contraindicated chemotherapy in Japan were excluded, which would expectedly decrease the potential impact of chemotherapy on the incidence of ILD exacerbation. Furthermore, after grouping the patients according to the presence or absence of sarcopenia, no significant difference in chemotherapy regimens had been noted, suggesting that sarcopenia can predict ILD exacerbations regardless of the type of anticancer drugs administered.

The reason for the involvement of sarcopenia in the ILD exacerbations and prognosis of lung cancer with ILD remains unclear. However, such involvement may be attributed to myokine, a bioactive substance secreted by skeletal muscle. Peroxisome proliferator-activated receptor y coactivator 1a (PGC-1a), a 
transcriptional coactivator required for the maintenance and generation of mitochondria, has been identified as an important factor in the relationship between exercise and inflammation ${ }^{31}$. Patients with sarcopenia have been found to have reduced PGC-1a in their skeletal muscle but enhanced interleukin-6 and tumor necrosis factor-a expression, causing inflammation and muscle proteolysis ${ }^{32,33}$. Previous studies have suggested that inflammation and undernutrition may contribute to the prognosis and exacerbation of ILD ${ }^{14,34-37}$. Based the aforementioned findings, anti-inflammatory treatment through myokine supplementation and maintenance of muscle mass through nutrition therapy and rehabilitation may be novel approaches for suppressing ILD exacerbation, as well as improving the prognosis for lung cancer with ILD.

At present, no treatment has been established for ILD exacerbation, which has shown high mortality rates $^{2,7,8}$. Therefore, patients with lung cancer and ILD often do not receive chemotherapy ${ }^{38,39}$. On the other hand, given evidence suggesting that chemotherapy may prolong survival, uniformly excluding these populations from chemotherapy may not be appropriate ${ }^{5,6}$. The present study showed that patients without sarcopenia were less prone to ILD exacerbation and could hence receive aggressive chemotherapy, which may contribute to a better prognosis. On the other hand, patients with sarcopenia may have a worse prognosis due to ILD exacerbation and hence require careful chemotherapy. In some cases, physicians may offer the patient the option to opt out from chemotherapy. Thus, the presence or absence of sarcopenia may be useful in determining treatment approaches for patients with lung cancer and ILD. Considering that these patients undergo whole-body CT for the purpose of staging evaluation, sarcopenia evaluation based on the psoas major muscle can be performed quite easily. Therefore, routine evaluation of sarcopenia upon introducing a chemotherapeutic drug may be better for patients with lung cancer and ILD.

The present study has several limitations worth noting. First, this was a single-center retrospective study with a relatively small sample size, which may introduce bias. However, lung cancer with ILD is a rare disease with a limited number of patients $3,4,9,10,12-14,38,40$. The number of patients included herein does not considerably differ from those included in other studies. Moreover, given the absence of large-scale phase III trials in patients with ILD-complicated lung cancer to date, evidence has been largely obtained from retrospective studies. Therefore, despite these limitations, we believe that the results presented herein are of considerable significance. Further studies are required to confirm our observations in other external validation cohorts. Second, diseases (e.g., diabetes, osteoporosis), drugs (e.g., statins, sulfonylureas, glinides, and antifibrotic drugs), and lifestyles (e.g., exercise and diet) that may affect skeletal muscle mass could not be completely assessed. On the other hand, the effects of sarcopenia due to complications can be considered minimal given that patients who had received steroids, had malignant tumors other than lung cancer, and had secondary ILD were excluded. Third, although the total psoas area was used, it might have been better to measure whole-body skeletal muscle mass using bioelectrical impedance or dual-energy X-ray absorption. However, these devices expose the patients to radiation and are considerably costly. Therefore, CT can be considered appropriate from the viewpoint of convenience. 
The present study showed that sarcopenia can predict ILD exacerbations and OS in patients with ILDcomplicated NSCLC who received chemotherapy. In the future, targeting sarcopenia can be expected to become a novel approach for suppressing ILD exacerbation and improving patient prognosis.

\section{Materials And Methods \\ 5.1. Patients}

Consecutive patients with non-small cell lung cancer (NSCLC) and pre-existing ILD who received platinum-based first-line chemotherapy at Tokyo Medical University Hospital between June 2010 and July 2020 were retrospectively analyzed. This study was approved by the institutional review board of Tokyo Medical University (permission No. TS2020-0207). This research was conducted in accordance with the 1964 Declaration of Helsinki and amendments. Due to retrospective nature of the study, the need of informed consent was waived by institutional review board of Tokyo Medical University. Our institution's official website had been used as a method to opt out from participation. All patients were cytologically and/or histologically diagnosed with NSCLC. The tumor, node, and metastasis (TNM) stage was evaluated based on the eighth edition of the TNM Classification of Lung Cancer ${ }^{41}$.

ILD was diagnosed when ground-glass attenuation and reticulation shadow or honeycombing with/without peripheral traction bronchiectasis were observed in both lung fields on chest HRCT. Diagnoses of chemotherapy-triggered ILD exacerbation were made in accordance with the following criteria detailed in previous studies: (1) deterioration of dyspnea; (2) new bilateral ground-glass attenuation and/or consolidation superimposed on pre-existing interstitial shadows; (3) ILD that could not be completely explained by pulmonary infectious disease, heart failure, or fluid overload; and (4) a less than 4-week interval between the last chemotherapy administration and onset of ILD exacerbation $9,10,13,14,42$.

The following patients were excluded: those (1) whose CT images did not include the spinous process at the third lumbar vertebral level, (2) who had other concomitant active malignancies, infectious diseases, or congested cardiac failure upon initial presentation, (3) who had insufficient clinical data, (4) who had been taking systemic steroids, (5) who had secondary ILD (i.e., interstitial pneumonia associated with connective tissue disease or antineutrophil cytoplasmic antibody-associated ILD), (6) who had received contraindicated chemotherapeutic drugs in Japan for patients with ILD (i.e., gemcitabine and epidermal growth factor receptor tyrosine kinase inhibitor), and (7) who received thoracic irradiation.

\subsection{Data collection}

Clinical information and follow-up data were obtained from patients' medical records. We collected data on patient demographics, age upon first-line treatment, sex, ECOG PS, TNM stage, histology, BMI, laboratory findings, pulmonary function test results, development of exacerbation ILD and mortality data. The follow-up period lasted until 31 July 2020. 


\subsection{Image analysis}

All patients underwent CT within 1 month before administering first-line chemotherapy. The PMI was measured at the third lumbar vertebral level on CT images using SYNAPSE VINCENT version 5.3.0001 software (Fujifilm Medical Co., Tokyo, Japan) with Hounsfield unit thresholds $(-29$ to +150$)$ in accordance with previous studies ${ }^{17-20}$ (Supplemental Fig. 1). All CT analyses were independently performed by trained pulmonologists (RK and HT) blinded to the patients' information, after which the obtained data were averaged. The PMI was calculated using the following formula: $\mathrm{PMI}\left(\mathrm{cm}^{2} / \mathrm{m}^{2}\right)=$ cross-sectional area of bilateral psoas muscles $\left(\mathrm{cm}^{2}\right) /$ height $\left(\mathrm{m}^{2}\right)$. PMl cutoff values for sarcopenia were defined as 6.36 and $3.92 \mathrm{~cm}^{2} / \mathrm{m}^{2}$ for males and females, respectively, based on a previous report defining sarcopenia in Asian adults ${ }^{28}$.

\subsection{Statistical analysis}

Data were described as numbers (percentages) or median (range). Categorical variables were analyzed using the chi-square test, whereas continuous variables were compared using the Mann-Whitney's $U$ test. Risk factors for chemotherapy-triggered exacerbation ILD were identified using univariate and multivariate logistic regression analyses. OS was defined as the time between initial chemotherapy and death or censoring. Patient survival was analyzed using the Kaplan-Meier method, after which differences were compared using the log-rank test. All statistical analyses were performed using SPSS software version 26.0 (IBM Corp., Armonk, NY), with $p<0.05$ indicating statistical significance.

\section{Declarations}

\section{Competing interests}

The authors declare no competing interests.

\section{Author contributions}

S. Abe and R. Kikuchi designed the research; S. Abe, R. Kikuchi, H. Takoi, M. Ishiwari, K. Toriyama, Y. Kono, and Y. Togashi analyzed the data; and S. Abe and R. Kikuchi wrote the paper.

\section{References}

1. Naccache, J. M. et al. Lung cancer and interstitial lung disease: a literature review. J. Thorac. Dis, 10, 3829-3844 (2018).

2. Ogura, T. et al. Summary of the Japanese Respiratory Society statement for the treatment of lung cancer with comorbid interstitial pneumonia. Respir. Investig, 57, 512-533 (2019).

3. Nagai, A., Chiyotani, A., Nakadate, T. \& Konno, K. Lung cancer in patients with idiopathic pulmonary fibrosis. Tohoku J. Exp. Med, 167, 231-237 (1992). 
4. Tomassetti, S. et al. The impact of lung cancer on survival of idiopathic pulmonary fibrosis. Chest, 147, 157-164 (2015).

5. Minegishi, Y. et al. The safety and efficacy of weekly paclitaxel in combination with carboplatin for advanced non-small cell lung cancer with idiopathic interstitial pneumonias. Lung Cancer, 71, 70-74 (2011).

6. Hanibuchi, M. et al. A multicenter, open-label, phase II trial of S-1 plus carboplatin in advanced nonsmall cell lung cancer patients with interstitial lung disease., 125, 93-99 (2018).

7. Lee, T. et al. Lung cancer in patients with idiopathic pulmonary fibrosis: clinical characteristics and impact on survival. Respir. Med, 108, 1549-1555 (2014).

8. Kinoshita, T. et al. Chemotherapy for non-small cell lung cancer complicated by idiopathic interstitial pneumonia. Oncol. Lett, 4, 477-482 (2012).

9. Kobayashi, H. et al. ILD-NSCLC-GAP index scoring and staging system for patients with non-small cell lung cancer and interstitial lung disease., 121, 48-53 (2018).

10. Enomoto, Y. et al. Low forced vital capacity predicts cytotoxic chemotherapy-associated acute exacerbation of interstitial lung disease in patients with lung cancer., 96, 63-67 (2016).

11. Nakao, S. et al. Chemotherapy-associated acute exacerbation of interstitial lung disease shortens survival especially in small cell lung cancer. Anticancer Res, 39, 5725-5731 (2019).

12. Kenmotsu, H. et al. The risk of cytotoxic chemotherapy-related exacerbation of interstitial lung disease with lung cancer. J. Thorac. Oncol, 6, 1242-1246 (2011).

13. Hamada, S. et al. Protective effect of bevacizumab on chemotherapy-related acute exacerbation of interstitial lung disease in patients with advanced non-squamous non-small cell lung cancer. $B M C$ Pulm. Med, 19, 72 (2019).

14. Kikuchi, R. et al. Glasgow Prognostic Score predicts chemotherapy-triggered acute exacerbationinterstitial lung disease in patients with non-small cell lung cancer. Thorac. Cancer, 12, 667-675 (2021).

15. Kikuchi, R. et al. Glasgow prognostic score for prediction of chemotherapy-triggered acute exacerbation interstitial lung disease in patients with small cell lung cancer. Thorac. Cancer, 12, 1681-1689 (2021).

16. Chen, L. K. et al. Sarcopenia in Asia: consensus report of the Asian Working Group for Sarcopenia. J. Am. Med. Dir. Assoc, 15, 95-101 (2014).

17. Nakamura, R. et al. Sarcopenia in resected NSCLC: effect on postoperative outcomes. J. Thorac. Oncol, 13, 895-903 (2018).

18. Shiroyama, T. et al. Impact of sarcopenia in patients with advanced non-small cell lung cancer treated with PD-1 inhibitors: a preliminary retrospective study. Sci. Rep, 9, 2447 (2019).

19. Nakada, T. et al. Risk factors and cancer recurrence associated with postoperative complications after thoracoscopic lobectomy for clinical stage I non-small cell lung cancer. Thorac. Cancer, 10, 1945-1952 (2019). 
20. Yang, M., Shen, Y., Tan, L. \& Li, W. Prognostic value of sarcopenia in lung cancer: a systematic review and meta-analysis. Chest, 156, 101-111 (2019).

21. Suzuki, Y. et al. Distinct profile and prognostic impact of body composition changes in idiopathic pulmonary fibrosis and idiopathic pleuroparenchymal fibroelastosis. Sci Rep, 8, 14074 (2018).

22. Moon, S. W. et al. Thoracic skeletal muscle quantification: low muscle mass is related with worse prognosis in idiopathic pulmonary fibrosis patients. Respir. Res, 20, 35 (2019).

23. Awano, N. et al. Quantitative computed tomography measures of skeletal muscle mass in patients with idiopathic pulmonary fibrosis according to a multidisciplinary discussion diagnosis: a retrospective nationwide study in Japan. Respir. Investig, 58, 91-101 (2020).

24. Suzuki, Y. et al. Cause of mortality and sarcopenia in patients with idiopathic pulmonary fibrosis receiving antifibrotic therapy. Respirology, 26, 171-179 (2021).

25. Nakano, A. et al. Early decrease in erector spinae muscle area and future risk of mortality in idiopathic pulmonary fibrosis. Sci. Rep, 10, 2312 (2020).

26. Pamoukdjian, F. et al. Prevalence and predictive value of pre-therapeutic sarcopenia in cancer patients: a systematic review. Clin. Nutr, 37, 1101-1113 (2018).

27. Cortellini, A. et al. Single-institution study of correlations between skeletal muscle mass, its density, and clinical outcomes in non-small cell lung cancer patients treated with first-line chemotherapy. Thorac. Cancer, 9, 1623-1630 (2018).

28. Hamaguchi, Y. et al. Proposal for new diagnostic criteria for low skeletal muscle mass based on computed tomography imaging in Asian adults. Nutrition, 32, 1200-1205 (2016).

29. Tsukagoshi, M. et al. Skeletal muscle mass predicts the outcome of nivolumab treatment for nonsmall cell lung cancer. Medicine, 99, e19059 (2020).

30. Shen, W. et al. Total body skeletal muscle and adipose tissue volumes: estimation from a single abdominal cross-sectional image. J. Appl. Physiol, 97, 2333-2338 (2004).

31. Handschin, C. \& Spiegelman, B. M. The role of exercise and PGC1 alpha in inflammation and chronic disease. Nature, 454, 463-469 (2008).

32. Arnold, A. S., Egger, A. \& Handschin, C. PGC-1a and myokines in the aging muscle - a mini-review. Gerontology, 57, 37-43 (2011).

33. Bowen, T. S., Schuler, G. \& Adams, V. Skeletal muscle wasting in cachexia and sarcopenia: molecular pathophysiology and impact of exercise training. J. Cachexia Sarcopenia Muscle, 6, 197-207 (2015).

34. Song, J. W., Hong, S. B., Lim, C. M., Koh, Y. \& Kim, D. S. Acute exacerbation of idiopathic pulmonary fibrosis: incidence, risk factors and outcome. Eur. Respir. J, 37, 356-363 (2011).

35. Zhuang, Y. et al. Incidence and impact of extra-pulmonary organ failures on hospital mortality in acute exacerbation of idiopathic pulmonary fibrosis. Sci. Rep, 10, 10742 (2020).

36. Zisman, D. A. et al. Serum albumin concentration and waiting list mortality in idiopathic interstitial pneumonia. Chest, 135, 929-935 (2009). 
37. Jouneau, S. et al. What are the best indicators to assess malnutrition in idiopathic pulmonary fibrosis patients? A cross-sectional study in a referral center. Nutrition, 62, 115-121 (2019).

38. Barczi, E. et al. Impact of interstitial lung disease and simultaneous lung cancer on therapeutic possibilities and survival. Thorac. Cancer, 11, 1911-1917 (2020).

39. Kawahara, T., Sakashita, H., Suzuki, T., Tateishi, T. \& Miyazaki, Y. Real world data of combined lung cancer and interstitial lung disease. J. Thorac. Dis, 11, 4144-4151 (2019).

40. Lee, K. J. et al. Prevalence, risk factors and survival of lung cancer in the idiopathic pulmonary fibrosis. Thorac. Cancer, 3, 150-155 (2012).

41. Goldstraw, P. et al. The IASLC Lung Cancer Staging Project: proposals for revision of the TNM stage groupings in the forthcoming (eighth) edition of the TNM Classification for Lung Cancer. J. Thorac. Oncol, 11, 39-51 (2016).

42. Collard, H. R. et al. Acute exacerbation of idiopathic pulmonary fibrosis. An international working group report. Am. J. Respir. Crit. Care Med, 194, 265-275 (2016).

\section{Figures}

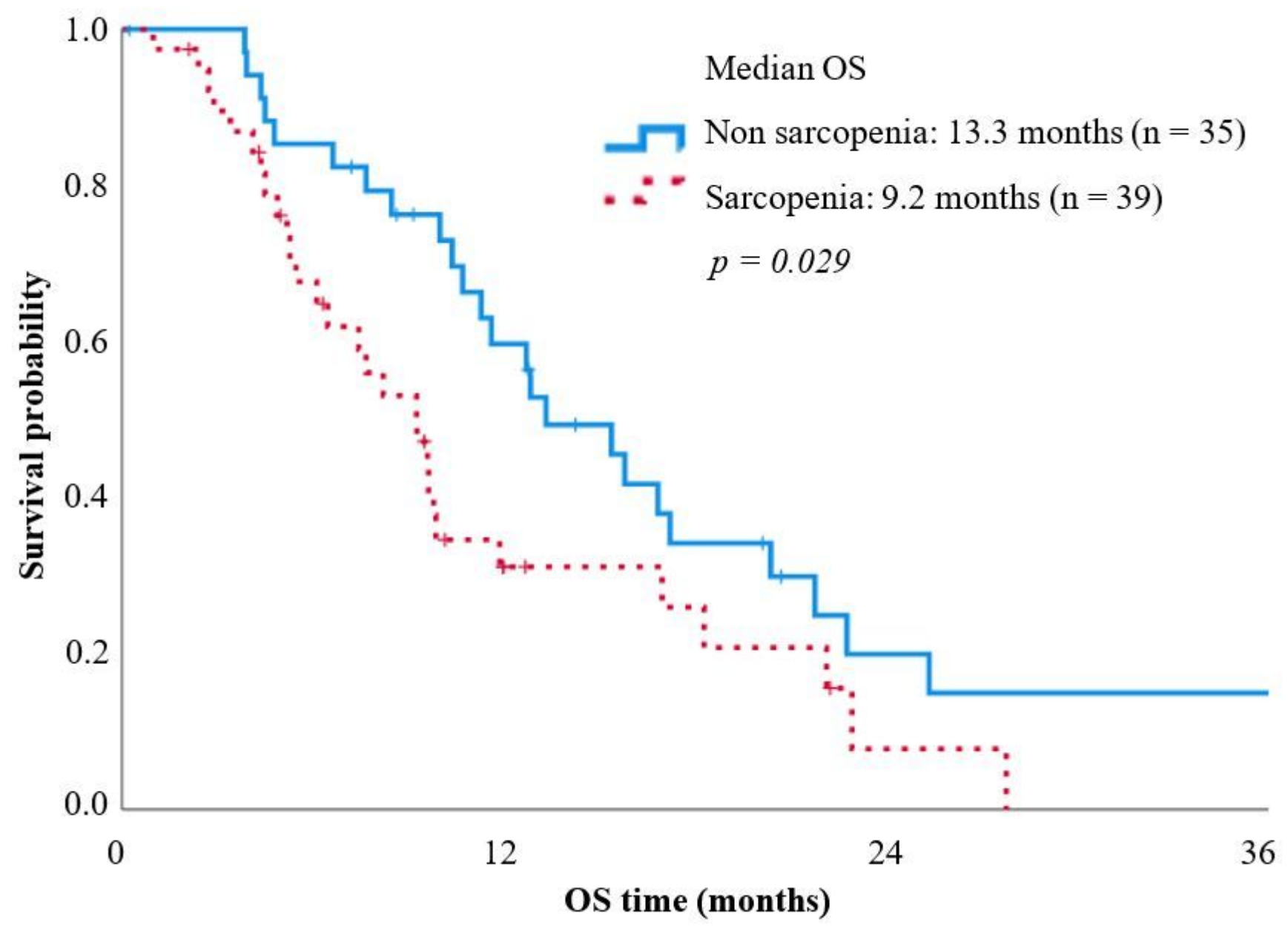




\section{Figure 1}

Kaplan-Meier curves showing the overall survival of patients who did $(n=39)$ and did not have sarcopenia $(n=35)$.

\section{Supplementary Files}

This is a list of supplementary files associated with this preprint. Click to download.

- Supportinginformation.docx 